\title{
Selective inhibition of soluble TNF using XPro1595 improves hippocampal pathology to promote improved neurological recovery following traumatic brain injury in mice
}

Kirsty Dixon ( $\sim$ kirsty.dixon@vcuhealth.org )

VCU: Virginia Commonwealth University

Katelyn Larson

Virginia Commonwealth University

Melissa Damon

Virginia Commonwealth University

Rajasa Randhi

Virginia Commonwealth University

Nancy Nixon-Lee

Virginia Commonwealth University

\section{Research Article}

Keywords: TNF, TNFR1, inflammation, glial reactivity, synaptic plasticity, learning and memory, depression, neuropathic pain

Posted Date: July 20th, 2021

DOl: https://doi.org/10.21203/rs.3.rs-734211/v1

License: (c) (i) This work is licensed under a Creative Commons Attribution 4.0 International License.

Read Full License 
1 Title: Selective inhibition of soluble TNF using XPro1595 improves hippocampal pathology to promote

2 improved neurological recovery following traumatic brain injury in mice

3

4 Katelyn Larson ${ }^{\text {a }}$, Melissa Damon ${ }^{\text {a }}$, Rajasa Randhi ${ }^{\text {a }}$, Nancy Nixon-Lee, Kirsty J. Dixon *

5

6 Department of Surgery, Virginia Commonwealth University, Richmond VA 23298

$7 \quad{ }^{a}$ equal contributors

$8 *$ corresponding author

9

10 Running Title: XPro1595 improves TBI outcomes

11 


\section{ABSTRACT}

BACKGROUND: Symptoms associated with traumatic brain injury (TBI) can be debilitating, and treatment without off-target side-effects remains a challenge. This study aimed to investigate the efficacy of selectively inhibiting the soluble form of TNF (solTNF) using the biologic XPro1595 in a mouse model of TBI. METHODS: Mild-to-moderate traumatic brain injury (CCI model) was induced in adult male C57B1/6J mice, with XPro1595 (10 mg/kg, S.C.) or vehicle being administered in a clinically relevant window (60 minutes post-injury). The animals were assessed for differences in neurological function, and hippocampal tissue was analyzed for inflammation and glial reactivity, as well as neuronal degeneration and plasticity. RESULTS: We report that unilateral CCI over the right parietal cortex in mice promoted deficits in learning and memory, depressive-like behavior and neuropathic pain. Using immunohistochemical and Western blotting techniques, we observed the cortical injury promoted a set of expected pathophysiology's within the hippocampus consistent with the observed neurological outcomes, including glial reactivity, enhanced neuronal dendritic degeneration (dendritic beading), and reduced synaptic plasticity (spine density and PSD-95 expression) within the DG and CA1 region of the hippocampus. These effects were prevented in the mice treated with XPro1595. CONCLUSION: Overall, we observed that selectively inhibiting solTNF using XPro1595 improved the pathophysiological and neurological sequelae of brain-injured mice, which provides support of its use in patients with TBI.

Keywords: TNF, TNFR1, inflammation, glial reactivity, synaptic plasticity, learning and memory, depression, neuropathic pain

\section{INTRODUCTION}

TBI affects $1.5 \mathrm{M}$ people annually in the United States, with approximately $80 \%$ being mild TBI, and symptoms range from physical, cognitive, psychological and/or psychosocial impairments. TBI is also a known risk factor for the later development of $\mathrm{AD}$ (1). The pathophysiological sequelae of TBI results from an acute and chronic phase of injury characterized by cell death and degeneration and neural 
connectome dysfunction/reorganization. In the acute phase, neuronal death is initiated by mechanical

forces, which alter cell membrane and vascular integrity, creating a microenvironment susceptible to

necrotic and apoptotic processes (2-5). The secondary injury phase occurs within minutes after the onset of TBI whereby the release of pro-inflammatory cytokines mobilizes immune and glial cells to the site of

42 injury causing edema and inflammation (6-11). This phase is also associated with gliosis, demyelination,

43 continued apoptosis and neuronal degeneration/plasticity. Within the damaged brain, the inflammatory

44 response causes a sustained upregulation of cytokines, such as interleukin 1-beta (IL-1 $\beta$ ), IL-6 and TNF

45 (12-14), and excess solTNF within the hippocampus activates TNFR1 to promote reductions in dendritic spine density, length, and protein expression (PSD-95, synapsin-1, GAP-43), modulating spine morphology and thus surface expression and synaptic localization of AMPA receptors, which temporarily suppresses LTP (15-23), all of which play a role in cognition, depression and neuropathic pain (18, 24-27).

Early clinical studies using TNF inhibitors (e.g. etanercept and infliximab) showed an inability to reduce mortality in sepsis patients $(28,29)$, which dampened enthusiasm for their use. More than 2 decades on,

52 additional meta-analysis' of data in sepsis patients revealed an overall improvement in survival rates (30),

53 when studies are sufficiently powered, which prompted a re-examination of their use. Subsequent studies

54 modulating TNF activity under injury and ischemic conditions have shown some promising outcomes in 55 both rodents and humans (31-35). Unfortunately, the TNF inhibitors have also shown an abundance of 56 side-effects, warranting FDA blackbox warnings regarding possible immunological dysfunction and heart 57 failure (36-38), and therefore the use of traditional TNF inhibitors should be cautioned in patients. These 58 side-effects are likely due to the differences in TNF receptor subtype functions, that have complicated the 59 TNF field until recently. TNF is a unique cytokine in that it is first produced as a transmembrane protein 60 (tmTNF) that preferentially activates TNF receptor 2 (TNFR2: CD120b or p75/p80) (39), but once cleaved 61 from the cell membrane TNF exists in a soluble form (solTNF) and preferentially activates TNF receptor 1 62 (TNFR1: CD120a or p55/p60) (39). Although both TNFR1 and TNFR2 can trigger some common 63 signaling pathways (40), TNFR2 activation generally promotes beneficial outcomes such as cell survival, 
64 induction of neurogenesis, and promotion of CNS autoimmunity (41-43), while TNFR1 activity generally

65 promotes detrimental outcomes such as cell death, aberrant neuronal plasticity, and exacerbation of the

66 existing inflammatory response $(41,44,45)$. The fact that traditional TNF inhibitors (e.g. etanercept and

67 infliximab) are unable to distinguish between the different TNF ligand or receptor subtypes, and thus

68 inhibiting TNFR2 activity, is likely driving the development of unwanted side-effects.

69

70 For these reasons, a novel 'second generation' TNF inhibitor was developed that selectively impedes the

71 activity of solTNF (XPro1595). XPro1595 has been successfully used in numerous pre-clinical

72 inflammatory disease models (46-50), with no known side-effects. It can effectively cross the BBB (51),

73 has a half-life of 19.1 hours (47), and can even improve heart function in a rodent model of cardiac arrest

74 (52). In recent clinical trials, XPro1595 was shown to be safe and well tolerated in cancer patients (53),

75 and interim data released from INmuneBio Inc. from a small clinical trial in Alzheimer's Disease patients

76 shows the biologic (used at $1.0 \mathrm{mg} / \mathrm{kg}$ S.C.) reverses brain white matter inflammation and CSF

77 inflammatory protein expression (54). Overlapping pathologies occur in brains following stroke, TBI and

78 those with Alzheimer's Disease, therefore, we sought to investigate whether using XPro1595 to selectively

79 bind and neutralize solTNF in a clinically relevant window following TBI in mice can improve 80 pathophysiological and functional outcomes.

81

\section{METHODS}

$83 \underline{\text { Animals }}$

84 Male C57B1/6J mice aged 2 to 4 months were used for the current study. Animals were housed in a 1285 hour light/dark cycle with food and water ad libitum. Procedures related to animal use were approved by 86 the Virginia Commonwealth University Institutional Animal Care and Use Committee (in accordance with $87 \quad$ NIH care and use of laboratory animals). 
The study groups included TBI-injured and sham-injured mice, treated with XPro1595 (XP) or vehicle (V),

91 that survived between 4 hours and 2 weeks. Animals surviving for 14 days underwent neurological testing,

92 prior to having their brain collected for analysis.

93

$94 \quad$ Traumatic Brain Injury Model

95 Mice were anaesthetized with ketamine $(75 \mathrm{mg} / \mathrm{kg}$ body weight) and xylazine ( $14 \mathrm{mg} / \mathrm{kg}$ body weight) by

96 intraperitoneal injection and positioned in a stereotaxic frame. A 5-mm craniotomy was made using a

97 portable drill over the right parietotemporal cortex $(-2.5 \mathrm{~mm}$ caudal and $2.0 \mathrm{~mm}$ lateral from bregma). A

98 drop of sterile saline was placed over the meninges to prevent it from drying out and tearing at the point of impact. A mild-to-moderate injury was generated (to avoid substantial structural damage to the underlying hippocampus) using a 3-mm beveled stainless-steel tip attached to an Impact One CCI device (Leica) at a velocity of $3.0 \mathrm{~m} / \mathrm{sec}$, depth of $0.5 \mathrm{~mm}$, and $150 \mathrm{msec}$ impact duration. Sham mice received anesthetic and skin incision only (since craniotomies themselves promote an inflammatory response). After CCI injury, the skin was sutured using 5-0 silk sutures. Starting 60 minutes post-injury, mice received twice weekly subcutaneous injections of XPro1595 (10 mg/kg in PBS, INmuneBio) or vehicle (0.1M PBS).

\section{Histological Preparation, Staining and Immunohistochemistry}

Mice were re-anaesthetized with ketamine $(75 \mathrm{mg} / \mathrm{kg})$ and xylazine $(14 \mathrm{mg} / \mathrm{kg})$ by intraperitoneal injection prior to transcardial perfusion or fresh brain dissection.

For qPCR analysis, fresh hippocampal tissue was dissected 6 hours post-injury (5 hours post-treatment), snap frozen in liquid nitrogen, and stored at $-80 \mathrm{oC}$. The frozen tissue was homogenized in RTL buffer (Qiagen) using lysing matrix D beads (MP Biomedicals). Total mRNA was extracted using RNeasy extraction kit (Qiagen) and converted to cDNA using reverse transcription kit (Applied Biosystems), followed by mRNA expression being processed using SYBRgreen real-time PCR (Applied Biosystems). Inflammatory cytokine expression for TNF, TNFR1, TNFR2, IL-6, and IL-1 $\beta$ was evaluated using Bio- 
Rad mouse primers. Values were normalized to GAPDH expression, and expressed as a fold change within

117 the ipsilateral tissue, as compared to the contralateral tissue.

For immunohistochemistry, transcardial perfusion was performed on WT and Thy1-YFPH mice, using approximately $15 \mathrm{ml}$ of PBS, followed by approximately $25 \mathrm{ml}$ of $4 \%$ paraformaldehyde (Sigma). The brain was dissected, stored in $4 \%$ paraformaldehyde for 2 hours, cryoprotected in $20 \%$ sucrose in PBS for

12248 hours, and then quickly frozen in OCT over isopentane on dry ice, and stored at $-80{ }^{\circ} \mathrm{C}$. Serial frozen 123 coronal sections were cut $40 \mu \mathrm{m}$ thick through the brain. Slides with sections from Thy1-YFPH mice containing hippocampus were washed in PBS to remove the OCT, and coverslipped in Prolong Gold Antifade mounting medium containing DAPI (Thermo Fisher). Slides with sections from WT mice containing hippocampus were permeabilized with $0.2 \%$ triton X-100 (Sigma) in $2 \%$ fish gel in PBS solution and immunohistochemically labelled with the primary antibody (1:2000 rabbit anti-GFAP, Dako; 1:2000 rabbit anti-IBA-1, Wako) overnight at $4{ }^{\circ} \mathrm{C}$. Sections were washed 3 times in PBS, incubated in fluorescent secondary antibodies (1:500, Molecular Probes) for $30 \mathrm{~min}$ at room temperature, washed an additional 3 times in PBS, and coverslipped in the same mounting medium containing DAPI (ThermoFisher). Sections from WT and Thy1-YFP mice were photographed at 60x oil with equal exposure on a Zeiss AxioImager Z2 microscope, connected to a Zeiss Monochrome digital camera (AxioCam MRm) with Zeiss Zen Software (Blue Edition version 1.1.2.0).

For Western blotting, fresh frozen hippocampal tissue will be homogenized according to Dixon laboratory protocols in RIPA lysis buffer, reduced and denatured using Invitrogen SDS loading buffer, and run on Invitrogen pre-cast bis-tris gradient gels, prior to being transferred to nitrocellulose membranes. Proteins of interest will be detected using antibodies to TNF, TNFR1, TNFR2, IL-6, IL-1beta, and IL-10, prior to incubation with secondary antibody, and then chemiluminescence visualized using the Bio-Rad ChemiDoc machine, and quantitating band measurement using Bio-Rad One optical density calculation. 
142 For Golgi labelling, transcardial perfusion was performed using approximately $15 \mathrm{ml}$ of PBS, followed by 143 approximately $25 \mathrm{ml}$ of $4 \%$ paraformaldehyde. The brain was dissected and prepared according to FD

144 Neurotechnology "Rapid Golgi stain” manufacturer's instructions. $100 \mu \mathrm{m}$ thick sagittal cryostat sections

145 were cut according to the manufacturer's instructions, and coverslipped in Permount mounting medium.

146 Golgi-stained sections containing hippocampus were scanned for dendrites at least $200 \mu \mathrm{m}$ in length in the

147 CA1 region. These dendrites were photographed at 100x oil on a Zeiss AxioImager Z2 microscope using

148 Köhler illumination, connected to a Q Imaging color digital camera (Model 2000R-F-CLR-12, 12-bit) with

149 Neurolucida software (version 2020.2.4).

150

$151 \quad$ Histological Analysis

152 Glial reactivity within the ipsilateral and contralateral cortex and hippocampus was determined by 153 quantitating GFAP and IBA-1 immunohistochemistry density, whereby images were converted to gray 154 scale and thresholded in NIH ImageJ software (version 1.52a) to identify the area fraction of pixels positive 155 for GFAP or IBA-1-immunoreactivity. Values for each photograph were averaged per section, per animal, 156 and then per group. To assess dendritic degeneration, photographs of DCX-positive dendrites from 157 immature neurons in the inner molecular layer of the dentate gyrus were quantitated for the presence (1) or 158 absence (0) of dendritic beadings/swellings. Values for each photograph were averaged per section, per 159 animal, and then per group. Subsequently, photographs of CA1 neurons from Thy1-YFP-H mice were 160 inspected for the presence of dendritic beadings. To assess dendritic spine density, photographs of CA1 161 hippocampus containing Golgi-stained dendrites were quantitated to determine dendritic spine density. The 162 number of dendritic spines along a dendritic segment $200 \mu \mathrm{m}$ in length were counted, and expressed as the 163 number of spines per $200 \mu \mathrm{m}$.

$165 \quad$ Neurological Testing

166 Morris Water Maze: Cognition (spatial learning and memory) was assessed on post-injury days 7 to 11 . A 167 blue plastic circular pool 5-foot wide and 11-inches deep was placed in the center of the room with large 
(3-foot wide) visual cues placed on 3 of the 4 surrounding walls. The pool was divided into 4 quadrants, arbitrarily nominated North, South, East, and West, and a glass beaker 5-inches wide was covered in cotton 170 gauze fabric and weighted upside down in the East quadrant of the pool. The mice were held by their tail 171 and placed into the pool facing the pool edge, and were given 4 trials per day, starting from each of the 4 172 quadrants for 5 consecutive days. Quadrant order on each consecutive day was as follows: NWES, WNSE,

173 EWSN, SEWN, NESW. The mice were allowed a maximum of 2 minutes to find the hidden platform, and

174 if unsuccessful were gently lifted onto the platform and allowed to remain there for 20 seconds before being 175 removed to their home cage. The number of quadrants the mice entered before reaching the platform, as 176 well as time taken to reach the platform were measured. On post-injury day 14 (72 hours after the last trial) 177 the mice underwent a probe trial, in which the platform from the East quadrant was removed from the pool, 178 the mice placed in the opposite quadrant (West quadrant), and allowed a maximum of 30 seconds to 'find' 179 the platform. Time spent in the East quadrant, and number of times entering the East quadrant were 180 measured.

182 Sucrose Preference Test: Depressive-like behavior was assessed prior to injury, and on post-injury days 3, 1837 and 14. Mice were housed individually for the duration of the test, and given free access to 2 water bottles 184 filled with either regular water or $2 \%$ sucrose diluted in water (Acros Organics Sucrose) overnight (5pm to 185 9am). The amount of regular water versus sucrose water that each mouse drank was measured. 186 Immediately after the test all mice were placed back into their group housing. Baseline testing was 187 performed on 3 separate evenings, and any mouse that did not have a pre-injury preference for the sucrose 188 solution was excluded from the analysis. The sucrose preference was calculated as the amount of sucrose 189 drunk as a percentage of total liquid drunk.

Mechanical von Frey Test: Pre-clinical rodent models of TBI display periorbital and hindpaw neuropathic 192 pain $(55,56)$. To assess the role of solTNF in the induction of injury-induced neuropathic pain, we 193 measured the level of mechanical hypersensitivity underneath the animals ipsilateral hindpaw 3-, 7- and 
14-days post-injury. In a dimly lit room, a 10" x 19" extension window screen (Thermwell) was fully extended and placed atop 2 polystyrene boxes, with a desk lamp placed behind and just under the height of the screen, angled towards the investigator. Four mice at a time were placed on top of the screen, with a

$197600 \mathrm{ml}$ glass beaker (Pyrex) placed over the top of each mouse to prevent escape. A disposable underpad 198 was draped over the beakers to minimize any light and/or movement stimulation. After 15 minutes 199 acclimatization under the beaker, hindpaw hypersensitivity was assessed by holding the von Frey filament 200 (Bioseb) handle under the screen, and slowly raising the end of the filament up through the screen to press 201 against the under-side of the mouse's hindpaw walking pad until a slight bend was observed in the fiber.

202 Continued advancement/bending of the filament does not necessarily produce more force of application. 203 The investigator tested the lightest filament first, and sequentially tested up through the filament sizes until 204 a positive result was established. A positive result was the mouse noticing 3 out of 5 consecutive tests for each filament, defined as the mouse withdrawing its foot, licking or shaking its foot, or rapidly moving its body away from the stimulus. Once a positive result was established for each mouse, the testing was

207 concluded for that mouse for that day. The testing occurred as rapidly as possible to reduce restraint 208 distress, although it was noticed that mice would often fall asleep during testing, which required gentle 209 tapping from underneath the screen to wake up the animal.

\section{$211 \quad$ Statistical Analysis}

212 All data were assessed for homogeneity of variance, after which statistical analysis was performed. 213 Histological differences were assessed using the Student's t-test, and behavioural differences (intra- and 214 inter-group analysis) were assessed using two-way repeated measures analysis of variance with Student215 Newman-Keuls method post hoc in SigmaPlot 13.0 where significance was $<0.05$. Data in figures are 216 expressed as mean \pm standard error of the mean.

\section{RESULTS}

219 Soluble TNF inhibition does not regulate IL-6 and IL-1 $\beta$ expression following TBI 
We sought to determine whether selective inhibition of solTNF following TBI regulates mRNA expression

221 of key inflammatory cytokines in select brain regions. We observed that neutralizing solTNF using

222 XPro1595 did not change the expression of the key pro-inflammatory cytokines IL-6 and IL-1 $\beta$ in the peri-

223 lesional cortex or ipsilateral hippocampus 6 hours following injury, compared to vehicle-treated injured

224 mice (Figure 1A\&B). Intriguingly, selective inhibition of solTNF significantly increased TNF expression

225 in the hippocampus following TBI (Figure 1C), with a tendency for increased production in the peri-lesional

226 region. We also examined TNFR1 expression, and observed a significant increase within the hippocampus

227 (Figure 1D). No significant differences were observed in TNFR2 expression, although trends suggest slight 228 increases in both hippocampus and peri-lesional region following injury (Figure 1E).

230 Soluble TNF inhibition reduces glial reactivity following TBI

231 To determine whether inhibiting solTNF attenuates injury-induced inflammation, mice were administered

232 XPro1595 (10 mg/kg S.C.) or vehicle, starting 60 minutes following the TBI. After a 2-week survival

233 period, we assessed glial reactivity by immunohistochemically labelling cortical and hippocampal tissue

234 with antibodies against GFAP (astrocytes) and IBA-1 (microglial/macrophage). Only minimal GFAP and

235 IBA-1 reactivity was detected in the uninjured hemisphere, independent of treatment (Figure 2A, B, G \&

$236 \mathrm{H}$ : contralateral hippocampus not shown). In comparison, GFAP is significantly upregulated in the cortical

237 peri-lesional region hippocampal CA1 region, which is significantly attenuated by treatment with XPro1595

238 (Figure 2A-F, M\&N). Injury also promoted an increase in IBA-1 expression in the cortical peri-lesional 239 and hippocampal CA1 regions (Figure 2G-L, O\&P), with XPro1595 treatment rescuing these effects back 240 to baseline levels.

$242 \quad$ Soluble TNF inhibition prevents hippocampal dendritic degeneration following TBI

243 Thy-1 YFPH mice display endogenous fluorescent labelling of hippocampal neurons (Figure 3A), which

244 in the CA1 regions of naïve mice exhibit long and wavy dendrites with an extensive number of post-synaptic

245 spines (Figure 3A\&B' and 3B' inset). Three days following TBI the dendrites appear disjointed, with 
extensive dendritic swellings (beading), and an absence of spines (Figure 3B' and 3B' inset). Using

247 immunohistochemistry to support the transgenic observations, we labeled hippocampal dendrites with

248 doublecortin (immature dendrites), and also observed extensive dendritic swellings in the mouse

249 hippocampal DG 14 days following TBI (Figure 3C); an affect that was significantly reduced in mice treated 250 with XPro1595 (Figure 3C\&D).

252 Soluble TNF inhibition prevents hippocampal dendritic plasticity following TBI

253 TBI was induced in mice and the expression of post-synaptic scaffolding protein PSD-95 was quantitated.

254 We observed that PSD-95 expression was reduced 3 days following injury (Figure 4A), but which was

255 prevented in injured mice treated with XPro1595. We next sought to determine whether our model of TBI

256 reduced the density of hippocampal CA1 dendritic spines, and whether soluble TNF inhibition can rescue

257 this effect. Mice were subjected to TBI and sacrificed 3 days later: a timepoint known to be consistent

258 with reduced hippocampal dendritic spine density. The spine density of CA1 hippocampal neurons

259 impregnated with Golgi stain was quantitated (Figure 5A). We observed that TBI reduced the density of 260 dendritic spines in the CA1 hippocampal region (Figure 5B), but was unaffected in the injured mice treated 261 with XPro1595 (Figure 5C).

262

263 Soluble TNF inhibition prevents TBI-induced functional deficits

264 Spatial Learning and Memory (Morris Water Maze): One of the most well-characterized neurological 265 symptoms associated with hippocampal injury is cognitive impairment, specifically learning and memory 266 (57). Therefore, we assessed the ability of mice to learn the MWM task for 5 consecutive days, on post267 injury days 7 to 11 . On the first day of testing the vehicle-treated injured mice were significantly less able 268 to find the platform than sham-injured mice, although XPro1595-treated injured mice able to find the 269 platform in the same amount of time as the sham-injured mice (Figure 5A). Notably, the vehicle-treated 270 injured mice were also significantly less able to find the MWM platform than XPro1595-treated mice. In 271 accordance with taking extra time to find the MWM platform, the vehicle-treated injured mice also 
272 entered significantly more MWM quadrants to find the platform compare to XPro1595-treated injured

273 mice (Figure 5B). Three days after the last MWM training day, the mice were tested in the MWM probe

274 test (removal of platform). The vehicle-treated injured mice spent significantly less time in the platform

275 quadrant compared to sham-injured mice (Figure 5C), and showed a strong tendency to spend less time in

276 the platform quadrant compared to XPro1595-treated injured mice. Similarly, the XPro1595-treated

277 injured mice also entered the platform quadrant more times than the vehicle-treated injured mice (Figure

278 5D).

279

280

Depressive-like Behavior (Sucrose Preference Test): In addition to cognitive impairment, depression is

281 frequently associated with aberrant hippocampal pathology following TBI. Therefore, we assessed the

282 development of depressive-like symptoms in brain-injured mice, using the sucrose preference test prior to

283 injury and on post-injury days 3, 7 and 14. Prior to injury the naïve uninjured untreated mice showed

284 approximately $90 \%$ preference for the sucrose solution (Figure 5E), but this was significantly reduced in

285 vehicle-treated mice acutely following injury, suggesting the CCI brain injury model promotes

286 depressive-like symptoms. Conversely, treating injured mice systemically with XPro1595 prevented a

287 reduction in their preference for the $2 \%$ sucrose solution, suggesting that XPro1595 affects the onset of

288 depressive-like symptoms and may be a useful clinical tool.

289

290

Hindpaw Neuropathic Pain (Mechanical von Frey Assay): Since depression and pain are frequently

291 comorbid following TBI, individuals with injury-induced chronic pain are at risk of developing dermal

292 mechanical and thermal hypersensitivity (58), we used the mechanical von Frey assay to assess hindpaw

293 hypersensitivity. Mice underwent testing prior to injury and on post-injury days 3, 7 and 14 . We

294 observed that all sham injured animals, independent of treatment, experienced a small increase in

295 hypersensitivity 3 days following injury (indicated by reduced mechanical threshold: Figure 5F), but this

296 was transient and quickly returned to baseline levels. In comparison, vehicle-treated injured mice were

297 significantly more hypersensitive on day 3 (almost a 3-fold increase compared to vehicle-treated sham 
mice), and this remained until the end of testing on day 14, suggesting these mice were in more pain,

299 which lasted longer than sham-operated mice. Conversely, XPro1595-treated injured mice experienced

300 less hypersensitivity than vehicle-treated injured mice (1.6-fold increase compared to XPro1595-treated

301 sham mice), and this quickly improved so that mice were no longer experiencing significantly greater

302 hypersensitivity at the end of testing. This suggests that XPro1595 therapy can reduce levels of pain

303 associated with brain injury.

\section{DISCUSSION}

306 Mild TBI is often associated with cognitive impairment, with many individuals experiencing significant 307 improvement over the first few months following an injury, although a non-trivial number may develop 308 persistent impairments. Importantly, cognitive impairment is strongly associated with the development of 309 depression, along with chronic pain, anxiety, and stress (18, 59-63). In individuals experiencing chronic 310 pain, symptoms often manifests as musculoskeletal pain (neck, shoulders, and back) and/or post-traumatic 311 headaches (64), many of whom also experience comorbid neuropathic pain (termed 'central pain') $(58,65)$.

313 The hippocampus plays an important role in regulating learning and memory, and structurally is composed 314 of a tri-synaptic neural circuit (66-68). This tri-synaptic circuit is composed of the performant pathway 315 projecting from the entorhinal cortex to the hippocampal DG, after which mossy fibers project from the DG 316 to synapse on pyramidal cells within the CA3 region, and then Schaffer collaterals project from the CA3 317 region to the CA1. Within this circuit, axon terminals synapse onto dendritic spines, releasing glutamate 318 from pre-synaptic terminals to activate AMPA receptors located within spines on the post-synaptic dendrite 319 to generate a miniature excitatory postsynaptic current (mEPSCs) that modulates efferent connectivity (i.e. 320 long-term potentiation, LTP) $(15,69)$. An individual's loss of memory is commonly known to be due to 321 damage anywhere along the hippocampal tri-synaptic neuronal circuit (70-72), but the hippocampus also 322 plays a role in the formation and retrieval of negative emotional memory, that is associated with pain and 323 depression $(18,20,27,73)$. Importantly, all of these symptoms share overlapping neuronal plasticity 
324 pathology, including reduced pre-synaptic puncta density, synaptic protein expression (PSD-95, synapsin-

325 1, GAP-43), and post-synaptic dendritic spine density (particularly mushroom spines), that collectively 326 inhibit LTP (excitatory synaptic connectivity) (17, 18, 60, 72, 74-79), but which also includes dendritic 327 degeneration in the form of dendritic "beads" or "swellings" $(72,76)$, likely due to blockade of protein and 328 mitochondrial trafficking. While TBI causes a sustained upregulation of cytokines, such as interleukin 1329 beta (IL-1 $\beta)$, IL-6 and TNF (12-14), TNF is unique in that its function is dependent upon activation of 330 receptor subtype (TNFR1 and TNFR2), and a strong connection exists between TNF/TNFR1-mediated 331 hippocampal plasticity, and impaired neurological outcomes $(18,25,27)$. Under physiologic conditions,

332 TNF regulates AMPA receptor surface expression (homeostatic synaptic plasticity), which subsequently regulates mEPSC currents and eventually LTP $(80,81)$, although excess production of solTNF under pathological conditions activates TNFR1 to promote reductions in dendritic spine density, length, and protein expression (PSD-95, synapsin-1, GAP-43), modulating spine morphology and surface expression and synaptic localization of AMPA receptors, which temporarily suppresses LTP to modulate cortical connectivity (15-23). Additionally, the induction of beading and degeneration causing impaired protein and mitochondrial transport along dendrites $(72,76,82,83)$, is also known to be induced by TNF (82). Therefore, selectively inhibiting solTNF/TNFR1 activity is critically important following TBI.

Following injury, TNF and TNFR1 expression increases, peaking between 4 and 24 hours post-injury (13, 45) (dependent on the animal model used (84)). Therefore, acute administration of XPro1595 (1 hour postinjury) in the current studies is not only clinically relevant, but is preventing activation of TNFR1 prior to the injury-induced peak of TNF/TNFR1 expression. Our observation that XPro1595 treatment does not change the expression pattern of the other key inflammatory cytokines within the hippocampus or perilesional region is important given that both IL1- $\beta$ and IL-6 are both pro- and anti-inflammatory following 347 trauma $(14,85)$. Reducing their expression may prevent their contribution to brain repair mechanisms, 348 while increasing their expression may exacerbate the extent of the injury. Interestingly, increases in TNF 349 and TNFR1 expression were observed in the XPro1595-treated injured mice, similar to antagonist induced 
receptor upregulation $(86,87)$, although this is functionally inconsequential given that the biologic

neutralizes any available solTNF present. Indeed, the increased TNF expression observed is likely

352 beneficial allowing for increased tmTNF/TNFR2 activity within the injury milieu. Importantly however,

353 XPro1595 treatment significantly reduced astrogliosis in all areas measured, as well as significantly

354 reducing cortical microglial reactivity (with a trend to reduce hippocampal microglial reactivity), when

355 measured two weeks post- injury. Therefore, it is plausible to suggest that despite TNF and TNFR1

356 upregulation, XPro1595 treatment reduced TNFR1 activity levels, although future studies investigating

357 TNFR1 downstream activities are needed to confirm this.

Intriguingly, we did not observe a significant upregulation of hippocampal microglial activation in this mild-to-moderate TBI model. Previous studies using the same injury model, but with a larger injury severity $(5 \mathrm{~m} / \mathrm{s}$ velocity) show robust microglial reactivity $(88)$, suggesting that this milder injury $(3 \mathrm{~m} / \mathrm{s}$ velocity) may not cause the same extent of hippocampal inflammation, since microglia are thought to be

364 None-the-less, injury-induced alterations in hippocampal pathology were observed, suggesting that even small levels of glial reactivity can promote detrimental changes to neuronal plasticity, including pathology 366 known to regulate cognitive impairment, depression and pain.

Although hippocampal synaptic plasticity (spine density) is known to occur in a bi-phasic manner with the greatest spine loss occurring within 2-3 days post-injury $(72,76)$ (our own data supports this notion), the 370 time-course of dendritic degeneration is less well characterized. Our data adds to the literature that initial 371 dendritic degeneration occurs within 3 days post-injury, and persists through to 14 days post-injury, even 372 being observed in newly developing immature dendrites (DCX-positive), thus the effects of even a mild373 to-moderate injury are long-lasting. Although some dendritic degeneration was observed in injured mice 374 treated with XPro1595, the amount of degeneration was significantly less, lending support for the beneficial 375 effects of XPro1595 administered acutely following TBI. 
377 Our study also assessed functional outcomes known to be associated with the hippocampus. The data shows

378 that injury-induced impairments in spatial learning (cognitive testing), as well as sucrose preference 379 (depressive-like behavior) and dermal hypersensitivity (neuropathic pain), the latter being clinically 380 relevant to the large number of individuals experiencing post-traumatic headaches. Since XPro1595 was 381 administered in a clinically relevant manner (subcutaneously), the ability of altered pathology in other brain 382 structures that may be contributing to the observed functional improvements following treatment cannot be 383 ruled out. None-the-less, the improvements in TBI pathology and associated functional outcomes, 384 combined with a lack of known side-effects in both animal models and patients, supports the use of 385 XPro1595 clinically in patients with TBI.

\section{CONCLUSION}

388 Excess levels of the inflammatory cytokine TNF play a prominent role in many inflammatory disease 389 pathologies, including the induction of aberrant pathology following a traumatic brain injury. Attempts to 390 use TNF receptor fusion proteins or monoclonal antibodies to regulate this cytokines function have shown 391 some successes clinically, but has been fraught with complications due to their numerous adverse side392 effects. Our data provide support for the clinical use of a novel "second generation" TNF inhibitor 393 XPro1595 that selectively inhibits only the detrimental soluble form of TNF to prevent the disease sequelae, 394 while sparing the beneficial transmembrane form of TNF to allow reparative cellular mechanisms to remain.

\section{ABBREVIATIONS}

397 LTP long term potentiation

$398 \quad$ solTNF $\quad$ soluble form of tumor necrosis factor

399 tmTNF transmembrane form of tumor necrosis factor

400 TNFR1 tumor necrosis factor receptor 1

401 TNFR2 tumor necrosis factor receptor 2 


\section{DECLARATIONS}

404 Ethics Approval and Consent to Participate

405 All experiments were performed under approval of the VCU Institutional Animal Care and Use

406 Committee.

407 Consent for Publication

408 Not applicable.

409 Availability of Data and Materials

410 The authors are always willing to collaborate and datasets generated and/or analyzed during the current

411 study are available from the corresponding author on reasonable request.

412 Competing Interests

413 The authors declare that they have no competing interests.

\section{$414 \quad$ Funding}

415 These studies were funded by a grant from the Virginia Commonwealth Neurotrauma Initiative (to KJD 416 (FP00001476)), and a donation of \$5,000 from Dr. Randall Merchant using MCVF funds (to KJD).

417 Microscopy was performed at the VCU Massey Cancer Center Microscopy Core Facility and supported, in 418 part, with funding from NIH-NCI Cancer Center Support Grant P30 CA016059. Funding bodies did not 419 contribute to the design of study or collection, analysis, interpretation of data or writing of the manuscript.

\section{Author's Contributions}

$421 \mathrm{KL}, \mathrm{MD}, \mathrm{RR}$ and NNL performed the experiments and tabulated the data. KJD designed, planned,

422 funded, analyzed and interpreted the data. All authors read and approved the final manuscript.

423 Acknowledgements

424 We thank Dr. Eleonora Mezzaroma for assisting with the qPCR protocol, and INmuneBio Inc. for providing 425 XPro1595 free of charge.

\section{REFERENCES}


1. Shively S, Scher Al, Perl DP, Diaz-Arrastia R. Dementia resulting from traumatic brain injury: what is the pathology? Arch Neurol. 2012;69(10):1245-51.

4302 2. Luo C, Jiang J, Lu Y, Zhu C. Spatial and temporal profile of apoptosis following lateral fluid 431 percussion brain injury. Chin J Traumatol. 2002;5(1):24-7.

432 3. Raghupathi R. Cell death mechanisms following traumatic brain injury. Brain Pathol. 433 2004;14(2):215-22.

434 4. O'Connor CA, Cernak I, Vink R. The temporal profile of edema formation differs between male and female rats following diffuse traumatic brain injury. Acta Neurochir Suppl. 2006;96:121-4.

5. Lotocki G, de Rivero Vaccari JP, Perez ER, Sanchez-Molano J, Furones-Alonso O, Bramlett HM, et al. Alterations in blood-brain barrier permeability to large and small molecules and leukocyte accumulation after traumatic brain injury: effects of post-traumatic hypothermia. I Neurotrauma. 2009;26(7):1123-34.

6. Morganti-Kossman MC, Lenzlinger PM, Hans V, Stahel P, Csuka E, Ammann E, et al. Production of cytokines following brain injury: beneficial and deleterious for the damaged tissue. Mol Psychiatry. 1997;2(2):133-6.

7. Ghirnikar RS, Lee YL, Eng LF. Inflammation in traumatic brain injury: role of cytokines and chemokines. Neurochem Res. 1998;23(3):329-40.

8. Lossinsky AS, Shivers RR. Structural pathways for macromolecular and cellular transport across the blood-brain barrier during inflammatory conditions. Review. Histol Histopathol. 2004;19(2):535-64.

9. Taupin P. Adult neurogenesis, neuroinflammation and therapeutic potential of adult neural stem cells. Int J Med Sci. 2008;5(3):127-32.

10. Ekmark-Lewen S, Lewen A, Israelsson C, Li GL, Farooque M, Olsson Y, et al. Vimentin and GFAP responses in astrocytes after contusion trauma to the murine brain. Restor Neurol Neurosci. 2010;28(3):311-21.

11. Homsi S, Piaggio T, Croci N, Noble F, Plotkine M, Marchand-Leroux C, et al. Blockade of acute microglial activation by minocycline promotes neuroprotection and reduces locomotor hyperactivity after closed head injury in mice: a twelve-week follow-up study. J Neurotrauma. 2010;27(5):911-21.

12. Yang SH, Gangidine M, Pritts TA, Goodman MD, Lentsch AB. Interleukin 6 mediates neuroinflammation and motor coordination deficits after mild traumatic brain injury and brief hypoxia in mice. Shock. 2013;40(6):471-5.

13. Lotocki G, Alonso OF, Dietrich WD, Keane RW. Tumor necrosis factor receptor 1 and its signaling intermediates are recruited to lipid rafts in the traumatized brain. J Neurosci. 2004;24(49):11010-6.

14. Murray KN, Parry-Jones AR, Allan SM. Interleukin-1 and acute brain injury. Front Cell Neurosci. 2015;9:18.

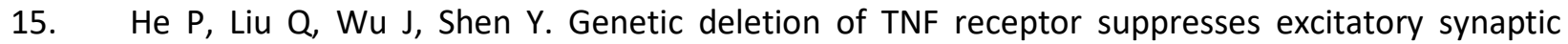
transmission via reducing AMPA receptor synaptic localization in cortical neurons. FASEB J. 2012;26(1):334-45.

16. Albensi BC, Mattson MP. Evidence for the involvement of TNF and NF-kappaB in hippocampal synaptic plasticity. Synapse. 2000;35(2):151-9.

17. Liu Y, Zhou L, Wang J, Li D, Ren WJ, Peng J, et al. TNF-alpha Differentially Regulates Synaptic Plasticity in the Hippocampus and Spinal Cord by Microglia-Dependent Mechanisms after Peripheral Nerve Injury. J Neurosci. 2017;37(4):871-81.

18. Dellarole A, Morton P, Brambilla R, Walters W, Summers S, Bernardes D, et al. Neuropathic paininduced depressive-like behavior and hippocampal neurogenesis and plasticity are dependent on TNFR1 signaling. Brain Behav Immun. 2014;41:65-81.

19. Mutso AA, Petre B, Huang L, Baliki MN, Torbey S, Herrmann KM, et al. Reorganization of hippocampal functional connectivity with transition to chronic back pain. J Neurophysiol. 2014;111(5):1065-76. 
20. Cardoso-Cruz H, Lima D, Galhardo V. Impaired spatial memory performance in a rat model of neuropathic pain is associated with reduced hippocampus-prefrontal cortex connectivity. J Neurosci. 2013;33(6):2465-80.

21. Chang Y, Yan LH, Zhang FK, Gong KR, Liu MG, Xiao Y, et al. Spatiotemporal characteristics of painassociated neuronal activities in primary somatosensory cortex induced by peripheral persistent nociception. Neurosci Lett. 2008;448(1):134-8.

22. Nakamura H, Katayama Y, Kawakami Y. Hippocampal CA1/subiculum-prefrontal cortical pathways induce plastic changes of nociceptive responses in cingulate and prelimbic areas. BMC Neurosci. 2010;11:100.

23. Becker D, Deller T, Vlachos A. Tumor necrosis factor (TNF)-receptor 1 and 2 mediate homeostatic synaptic plasticity of denervated mouse dentate granule cells. Sci Rep. 2015;5:12726.

24. Hennessy E, Gormley S, Lopez-Rodriguez AB, Murray C, Murray C, Cunningham C. Systemic TNFalpha produces acute cognitive dysfunction and exaggerated sickness behavior when superimposed upon progressive neurodegeneration. Brain Behav Immun. 2017;59:233-44.

25. Postal M, Lapa AT, Sinicato NA, de Oliveira Pelicari K, Peres FA, Costallat LT, et al. Depressive symptoms are associated with tumor necrosis factor alpha in systemic lupus erythematosus. J Neuroinflammation. 2016;13:5.

26. Gerard E, Spengler RN, Bonoiu AC, Mahajan SD, Davidson BA, Ding H, et al. Chronic constriction injury-induced nociception is relieved by nanomedicine-mediated decrease of rat hippocampal tumor necrosis factor. Pain. 2015;156(7):1320-33.

27. Martuscello RT, Spengler RN, Bonoiu AC, Davidson BA, Helinski J, Ding $H$, et al. Increasing TNF levels solely in the rat hippocampus produces persistent pain-like symptoms. Pain. 2012;153(9):1871-82. 28. Grau GE, Maennel DN. TNF inhibition and sepsis -- sounding a cautionary note. Nat Med. 1997;3(11):1193-5.

29. Fisher CJ, Jr., Agosti JM, Opal SM, Lowry SF, Balk RA, Sadoff JC, et al. Treatment of septic shock with the tumor necrosis factor receptor:Fc fusion protein. The Soluble TNF Receptor Sepsis Study Group. N Engl J Med. 1996;334(26):1697-702.

30. Qiu P, Cui X, Sun J, Welsh J, Natanson C, Eichacker PQ. Antitumor necrosis factor therapy is associated with improved survival in clinical sepsis trials: a meta-analysis. Crit Care Med. 2013;41(10):2419-29.

31. Tobinick E, Kim NM, Reyzin G, Rodriguez-Romanacce H, DePuy V. Selective TNF inhibition for chronic stroke and traumatic brain injury: an observational study involving 629 consecutive patients treated with perispinal etanercept. CNS Drugs. 2012;26(12):1051-70.

32. Tobinick E, Rodriguez-Romanacce H, Levine A, Ignatowski TA, Spengler RN. Immediate neurological recovery following perispinal etanercept years after brain injury. Clin Drug Investig. 2014;34(5):361-6.

33. Baratz R, Tweedie D, Rubovitch V, Luo W, Yoon JS, Hoffer BJ, et al. Tumor necrosis factor-alpha synthesis inhibitor, 3,6'-dithiothalidomide, reverses behavioral impairments induced by minimal traumatic brain injury in mice. J Neurochem. 2011;118(6):1032-42.

34. Chio $\mathrm{CC}$, Chang $\mathrm{CH}$, Wang $\mathrm{CC}$, Cheong $\mathrm{CU}$, Chao $\mathrm{CM}$, Cheng $\mathrm{BC}$, et al. Etanercept attenuates traumatic brain injury in rats by reducing early microglial expression of tumor necrosis factor-alpha. BMC Neurosci. 2013;14:33.

35. Chio CC, Lin JW, Chang MW, Wang CC, Kuo JR, Yang CZ, et al. Therapeutic evaluation of etanercept in a model of traumatic brain injury. J Neurochem. 2010;115(4):921-9.

36. Kwon HJ, Cote TR, Cuffe MS, Kramer JM, Braun MM. Case reports of heart failure after therapy with a tumor necrosis factor antagonist. Ann Intern Med. 2003;138(10):807-11. 
37. Thomas SS, Borazan N, Barroso N, Duan L, Taroumian S, Kretzmann B, et al. Comparative Immunogenicity of TNF Inhibitors: Impact on Clinical Efficacy and Tolerability in the Management of Autoimmune Diseases. A Systematic Review and Meta-Analysis. BioDrugs. 2015;29(4):241-58.

38. Maneiro JR, Salgado E, Gomez-Reino JJ. Immunogenicity of monoclonal antibodies against tumor necrosis factor used in chronic immune-mediated Inflammatory conditions: systematic review and metaanalysis. JAMA Intern Med. 2013;173(15):1416-28.

39. Fischer R, Marsal J, Gutta C, Eisler SA, Peters N, Bethea JR, et al. Novel strategies to mimic transmembrane tumor necrosis factor-dependent activation of tumor necrosis factor receptor 2 . Sci Rep. 2017;7(1):6607.

40. Wajant H, Siegmund D. TNFR1 and TNFR2 in the Control of the Life and Death Balance of Macrophages. Front Cell Dev Biol. 2019;7:91.

41. Longhi L, Perego C, Ortolano F, Aresi S, Fumagalli S, Zanier ER, et al. Tumor necrosis factor in traumatic brain injury: effects of genetic deletion of p55 or p75 receptor. J Cereb Blood Flow Metab. 2013;33(8):1182-9.

42. Faustman DL, Davis M. TNF Receptor 2 and Disease: Autoimmunity and Regenerative Medicine. Front Immunol. 2013;4:478.

43. Atretkhany KN, Mufazalov IA, Dunst J, Kuchmiy A, Gogoleva VS, Andruszewski D, et al. Intrinsic TNFR2 signaling in T regulatory cells provides protection in CNS autoimmunity. Proc Natl Acad Sci U S A. 2018;115(51):13051-6.

44. Yang J, You Z, Kim HH, Hwang SK, Khuman J, Guo S, et al. Genetic analysis of the role of tumor necrosis factor receptors in functional outcome after traumatic brain injury in mice. J Neurotrauma. 2010;27(6):1037-46.

45. Knoblach SM, Fan L, Faden Al. Early neuronal expression of tumor necrosis factor-alpha after experimental brain injury contributes to neurological impairment. J Neuroimmunol. 1999;95(1-2):115-25. 46. Brambilla R, Ashbaugh JJ, Magliozzi R, Dellarole A, Karmally S, Szymkowski DE, et al. Inhibition of soluble tumour necrosis factor is therapeutic in experimental autoimmune encephalomyelitis and promotes axon preservation and remyelination. Brain. 2011;134(Pt 9):2736-54.

47. Clausen BH, Degn M, Martin NA, Couch Y, Karimi L, Ormhoj M, et al. Systemically administered anti-TNF therapy ameliorates functional outcomes after focal cerebral ischemia. J Neuroinflammation. 2014;11:203.

48. MacPherson KP, Sompol P, Kannarkat GT, Chang J, Sniffen L, Wildner ME, et al. Peripheral administration of the soluble TNF inhibitor XPro1595 modifies brain immune cell profiles, decreases betaamyloid plaque load, and rescues impaired long-term potentiation in 5xFAD mice. Neurobiol Dis. 2017;102:81-95.

49. Cavanagh C, Tse YC, Nguyen HB, Krantic S, Breitner JC, Quirion R, et al. Inhibiting tumor necrosis factor-alpha before amyloidosis prevents synaptic deficits in an Alzheimer's disease model. Neurobiol Aging. 2016;47:41-9.

50. Sama DM, Mohmmad Abdul H, Furman JL, Artiushin IA, Szymkowski DE, Scheff SW, et al. Inhibition of soluble tumor necrosis factor ameliorates synaptic alterations and $\mathrm{Ca} 2+$ dysregulation in aged rats. PLoS One. 2012;7(5):e38170.

51. Karamita M, Barnum C, Mobius W, Tansey MG, Szymkowski DE, Lassmann H, et al. Therapeutic inhibition of soluble brain TNF promotes remyelination by increasing myelin phagocytosis by microglia. JCl Insight. 2017;2(8).

52. Wang C, Yang J, Z. H, Guo Q, Wu X, Bradley J, et al., editors. XPro1595 reduces the severity of post resuscitation myocardial dysfunction in a rat model of cardiac arrest. Circulation; 2018.

53. INmuneBiolnc. INmune Bio Announces Final Phase I Clinical Data for its Soluble TNF Inhibitor, INB03, Demonstrates Efficacy and Safety; INB03 is Advancing to Phase II Trials. https://wwwglobenewswirecom/news-release/2019/12/17/1961610/0/en/INmune-Bio-Announces- 
Final-Phase-I-Clinical-Data-for-its-Soluble-TNF-Inhibitor-INB03-Demonstrates-Efficacy-and-Safety-INB03is-Advancing-to-Phase-II-Trialshtml. 2019.

572 54. INmuneBiolnc. INmune Bio, Inc. Announces XPro1595 Found to Decrease Neuroinflammation and 573 Neurodegeneration Biomarkers in Patients with Alzheimer's Disease in Phase $1 \mathrm{~b}$ Trial https://www.globenewswire.com/news-release/2021/01/21/2162007/0/en/INmune-Bio-IncAnnounces-XPro1595-Found-to-Decrease-Neuroinflammation-and-Neurodegeneration-Biomarkers-inPatients-with-Alzheimer-s-Disease-in-Phase-1b-Trial.html2021 [

55. Elliott MB, Oshinsky ML, Amenta PS, Awe OO, Jallo Jl. Nociceptive neuropeptide increases and periorbital allodynia in a model of traumatic brain injury. Headache. 2012;52(6):966-84.

56. Macolino CM, Daiutolo BV, Albertson BK, Elliott MB. Mechanical alloydnia induced by traumatic brain injury is independent of restraint stress. J Neurosci Methods. 2014;226:139-46.

57. Akhondzadeh S. Hippocampal synaptic plasticity and cognition. J Clin Pharm Ther. 1999;24(4):2418.

58. Ofek H, Defrin R. The characteristics of chronic central pain after traumatic brain injury. Pain. 2007;131(3):330-40.

59. Sullivan-Singh SJ, Sawyer K, Ehde DM, Bell KR, Temkin N, Dikmen S, et al. Comorbidity of pain and depression among persons with traumatic brain injury. Arch Phys Med Rehabil. 2014;95(6):1100-5.

60. Qiao H, Li MX, Xu C, Chen HB, An SC, Ma XM. Dendritic Spines in Depression: What We Learned from Animal Models. Neural Plast. 2016;2016:8056370.

61. Bay E, Kirsch N, Gillespie B. Chronic stress conditions do explain posttraumatic brain injury depression. Res Theory Nurs Pract. 2004;18(2-3):213-28. alpha inhibitor) decreases depression and anxiety-like behaviour in rat model of chronic mild stress. Basic Clin Pharmacol Toxicol. 2013;112(5):335-40.

63. Grandhi R, Tavakoli S, Ortega C, Simmonds MJ. A Review of Chronic Pain and Cognitive, Mood, and Motor Dysfunction Following Mild Traumatic Brain Injury: Complex, Comorbid, and/or Overlapping Conditions? Brain Sci. 2017;7(12).

64. Nampiaparampil DE. Prevalence of chronic pain after traumatic brain injury: a systematic review. JAMA. 2008;300(6):711-9.

599 65. Defrin R, Gruener H, Schreiber S, Pick CG. Quantitative somatosensory testing of subjects with chronic post-traumatic headache: implications on its mechanisms. Eur J Pain. 2010;14(9):924-31.

66. Kumar RG, Gao S, Juengst SB, Wagner AK, Fabio A. The effects of post-traumatic depression on cognition, pain, fatigue, and headache after moderate-to-severe traumatic brain injury: a thematic review. Brain Inj. 2018;32(4):383-94.

67. Phillips KF, Deshpande LS. Repeated low-dose organophosphate DFP exposure leads to the development of depression and cognitive impairment in a rat model of Gulf War Illness. Neurotoxicology. 2016;52:127-33.

68. Grzegorski T, Losy J. Cognitive impairment in multiple sclerosis - a review of current knowledge and recent research. Rev Neurosci. 2017;28(8):845-60.

69. Kusters R, Kapitein LC, Hoogenraad CC, Storm C. Shape-induced asymmetric diffusion in dendritic spines allows efficient synaptic AMPA receptor trapping. Biophys J. 2013;105(12):2743-50.

70. Mahmmoud RR, Sase S, Aher YD, Sase A, Groger M, Mokhtar M, et al. Spatial and Working Memory Is Linked to Spine Density and Mushroom Spines. PLoS One. 2015;10(10):e0139739.

71. Leuner B, Gould E. Structural plasticity and hippocampal function. Annu Rev Psychol. 2010;61:111-40, C1-3.

72. Gao X, Deng P, Xu ZC, Chen J. Moderate traumatic brain injury causes acute dendritic and synaptic degeneration in the hippocampal dentate gyrus. PLoS One. 2011;6(9):e24566. 
73. Fasick V, Spengler RN, Samankan S, Nader ND, Ignatowski TA. The hippocampus and TNF: Common links between chronic pain and depression. Neurosci Biobehav Rev. 2015;53:139-59.

74. Yan BC, Park JH, Ahn JH, Lee JC, Won MH, Kang IJ. Postsynaptic density protein (PSD)-95 expression is markedly decreased in the hippocampal CA1 region after experimental ischemia-reperfusion injury. J Neurol Sci. 2013;330(1-2):111-6.

75. Wakade C, Sukumari-Ramesh S, Laird MD, Dhandapani KM, Vender JR. Delayed reduction in hippocampal postsynaptic density protein-95 expression temporally correlates with cognitive dysfunction following controlled cortical impact in mice. J Neurosurg. 2010;113(6):1195-201. hippocampal CA1 field following traumatic brain injury. J Neurotrauma. 2005;22(7):719-32.

77. Ansari MA, Roberts KN, Scheff SW. Oxidative stress and modification of synaptic proteins in hippocampus after traumatic brain injury. Free Radic Biol Med. 2008;45(4):443-52.

78. Ren WJ, Liu Y, Zhou LJ, Li W, Zhong Y, Pang RP, et al. Peripheral nerve injury leads to working memory deficits and dysfunction of the hippocampus by upregulation of TNF-alpha in rodents. Neuropsychopharmacology. 2011;36(5):979-92.

79. Winston CN, Chellappa D, Wilkins T, Barton DJ, Washington PM, Loane DJ, et al. Controlled cortical impact results in an extensive loss of dendritic spines that is not mediated by injury-induced amyloid-beta accumulation. J Neurotrauma. 2013;30(23):1966-72.

80. Stellwagen D, Malenka RC. Synaptic scaling mediated by glial TNF-alpha. Nature. 2006;440(7087):1054-9.

81. Becker D, Zahn N, Deller T, Vlachos A. Tumor necrosis factor alpha maintains denervation-induced homeostatic synaptic plasticity of mouse dentate granule cells. Front Cell Neurosci. 2013;7:257.

82. Kim JY, Shen S, Dietz K, He Y, Howell O, Reynolds R, et al. HDAC1 nuclear export induced by pathological conditions is essential for the onset of axonal damage. Nat Neurosci. 2010;13(2):180-9.

83. Greenwood SM, Mizielinska SM, Frenguelli BG, Harvey J, Connolly CN. Mitochondrial dysfunction and dendritic beading during neuronal toxicity. J Biol Chem. 2007;282(36):26235-44.

84. Woodcock T, Morganti-Kossmann MC. The role of markers of inflammation in traumatic brain injury. Front Neurol. 2013;4:18.

85. Scheller J, Chalaris A, Schmidt-Arras D, Rose-John S. The pro- and anti-inflammatory properties of the cytokine interleukin-6. Biochim Biophys Acta. 2011;1813(5):878-88.

86. Yoburn BC, Billings B, Duttaroy A. Opioid receptor regulation in mice. J Pharmacol Exp Ther. 1993;265(1):314-20.

87. Wonnacott $\mathrm{S}$. The paradox of nicotinic acetylcholine receptor upregulation by nicotine. Trends Pharmacol Sci. 1990;11(6):216-9.

651 88. Dixon KJ, Theus MH, Nelersa CM, Mier J, Travieso LG, Yu TS, et al. Endogenous neural 652 stem/progenitor cells stabilize the cortical microenvironment after traumatic brain injury. J Neurotrauma. 653 2015;32(11):753-64.

654

\section{FIGURE LEGENDS}

Figure 1: Pro-inflammatory cytokine expression 6 hours following TBI. Graphs show the fold change

657 of expression within the ipsilateral tissue, as compared to the contralateral tissue of IL-6 (A), IL-1 $\beta$ (B), 
significantly increased TNF and TNFR1 expression within the hippocampus, with a tendency for increased expression of TNFR2. Hipp=Hippocampal; V=Vehicle; XP=XPro1595; TBI+Veh = white bars; TBI+XP $662=$ grey bars; $\mathrm{n}=4$ per group; $* \mathrm{p}<0.05$.

Figure 2: Glial reactivity in the peri-lesional cortex and hippocampus two weeks following TBI. Negligible GFAP (astrocytes) and IBA-1 (microglia/macrophages) protein is expressed in the uninjured cortex (A,B,G\&H), and hippocampus (images not shown), independent of treatment. TBI promotes gliosis in both the peri-lesional region and hippocampus (C,E,I\&K), although this is prevented by using XPro1595 to neutralize soluble TNF (D, F, J \& L). Graphs show semi-quantitation of glial reactivity in the ipsilateral and contralateral hemisphere following TBI (M-P). $\mathrm{C}-\mathrm{V}=$ contralateral hemisphere+vehicle; $\mathrm{C}-\mathrm{XP}=$ contralateral hemisphere+XPro1595; I-V = ipsilateral hemisphere+vehicle; I-XP = ipsilateral hemisphere+ XPro1595; TBI+V n=9, TBI+XP n=9; ** $=\mathrm{p}<0.01, * * *=p<0.001$. Scalebar in $\mathrm{L}=50 \mu \mathrm{m}$.

672

Figure 3: Hippocampal dendritic degeneration following CCI injury. Thy1-YFP-labelled mouse

674 hippocampus showing dentate gyrus (DG), CA1, CA2 and CA3 regions (A). Neuronal dendrites from CA1 region in naive mice show normal morphology (B'), while 3 days post-injury CA1 dendrites develop abnormalities (B')). CCI-injury appears to reduce spine density, and promotes swellings (beading) along the length of the dendrite (B). In naïve uninjured animals, dendrites from immature neurons within the 678 hippocampal DG that are immunoreactive for doublecortin, show an absence of dendritic swellings (C'). 679 However, extensive swellings are observed on immature dendrites of the DG 14 days following the injury 680 (C',) that can be significantly reduced when XPro1595 is administered starting 60 minutes post-injury 681 (C',\&D). V=Vehicle; XP=XPro1595; TBI+V n=4, TBI+XP n=4; * = p<0.05; Scalebar in B = $20 \mu \mathrm{m}, \mathrm{B}$ 682 inset $=5 \mu \mathrm{m}$, and $\mathrm{C}=2 \mu \mathrm{m}$. hippocampus was semi-quantitated for PSD-95 expression; a prominent post-synaptic scaffolding protein, 
3 days post-CCI injury. Western blot analysis reveals injury reduced hippocampal PSD-95 expression (A), which was prevented in mice treated with XPro1595 one-hour post-injury.

(B) Morphology of Golgistained hippocampal slices from sham- and CCI-injured mice, treated with either XPro1595 or Vehicle show changes in spine density. Golgi-stained dendrites were quantitated for spine density (number of spines on a dendrite $200 \mu \mathrm{m}$ in length), revealing that injury reduces dendritic spine density (C), which is prevented in the XPro1595-treated injured mice. Veh=Vehicle, XP=XPro1595; (A) n=3 per group, (C) Sham+Veh n=7, Sham $+X P n=5, T B I+V e h n=10, T B I+X P n=10 ; * *=p<0.01$. Scalebar in $B=2 \mu m$.

Figure 5: TBI-induced solTNF promotes hippocampal-associated functional impairments. Sham- or CCI-injured mice, treated with vehicle or XPro1595 (starting 60 minutes post-injury) were assessed on spatial learning and memory in the Morris Water Maze (MWM) for 5 consecutive days (post-injury days 7-11). (A\&B) The vehicle-treated injured mice took significantly longer on day 1 to learn to find the platform, by entering significantly more quadrants. Conversely, XPro1595-treated injured mice were able to find the platform in the same amount of time, entering the same number of quadrants, compared to shaminjured mice (independent of treatment). (C\&D) Three days after the last training session (14 days postinjury) the mice were assessed on remembering the location of the platform (probe test: platform removed). Mice with TBI spent less time in the platform quadrant than sham-injured mice, and a tendency to enter the platform quadrant less than sham-injured mice. XPro1595-treated injured mice spent an equal amount of time in the platform quadrant compared to sham-injured mice, and an equal amount of entries into the platform quadrant. (E) Mice were assessed using the sucrose preference test prior to injury (baseline), and on post-injury days 3,7 and 14 . Prior to injury the mice preferred to drink the $2 \%$ sucrose water approximately $90 \%$ of the time. Comparatively, vehicle-treated injured mice displayed a temporary reduction in their preference for the sucrose water, which was not observed in the XPro1595-treated injured mice. (F) Mice were assessed on their level of hindpaw mechanical hypersensitivity prior to injury

710 (baseline), and on post-injury days 3, 7 and 14. The sham-injured mice displayed a significant increase in 711 hypersensitivity (reduced threshold) 3 days post-injury, that returns to baseline after the first week. 
712 Conversely, the vehicle-treated injured mice display increased hypersensitivity that persists until the end of 713 testing at post-injury day 14 . While the XPro1595-treated injured mice display significantly more 714 hypersensitivity 3 days post-injury, this effect is only temporary, similar to the sham-injured mice. Veh= 715 vehicle; XP = XPro1595; (A-D) Sham+Veh n=13, Sham+XP n=12, TBI+Veh n=15, TBI+XP n=14; (E) 716 TBI+Veh n=4, TBI+XP n=5; (F) Sham+Veh n=10, Sham+XP n=11, TBI+Veh n=5, TBI+XP n=4; * = $717 \mathrm{p}<0.05, * *=\mathrm{p}<0.01, * * *=\mathrm{P}<0.001$ 


\section{Figures}
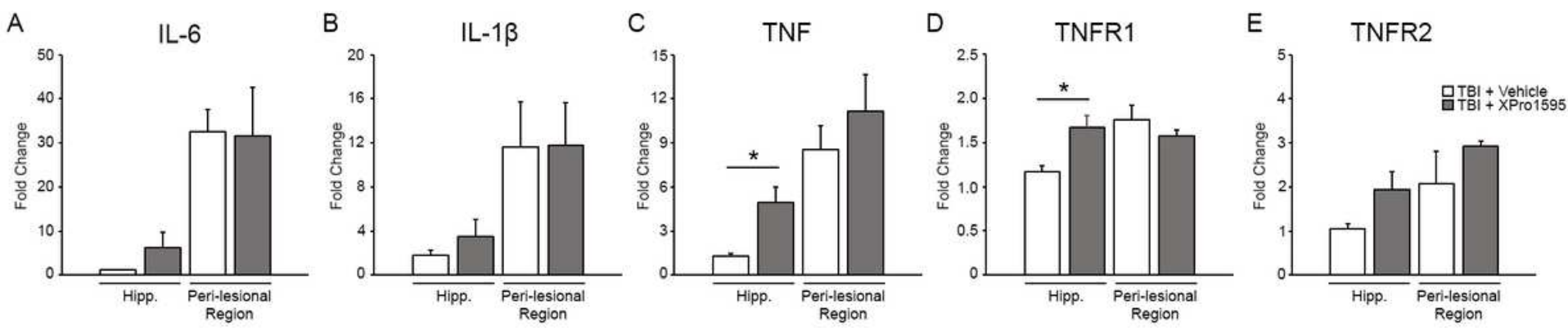

\section{Figure 1}

Pro-inflammatory cytokine expression 6 hours following TBI. Graphs show the fold change of expression within the ipsilateral tissue, as compared to the contralateral tissue of IL-6 (A), IL-1 $\beta$ (B), TNF (C), TNFR1 (D), and TNFR2 (E), with either vehicle or XPro1595 treatment. qPCR analysis reveals selective inhibition of soluble TNF does not alter IL-6 or IL-1 $\beta$ expression. In contract, XPro1595 significantly increased TNF and TNFR1 expression within the hippocampus, with a tendency for increased 660 expression of TNFR2. Hipp=Hippocampal; V=Vehicle; XP=XPro1595; TBI+Veh = white bars; TBI+XP 661 = grey bars; $n=4$ per group; * $p<0.05$.
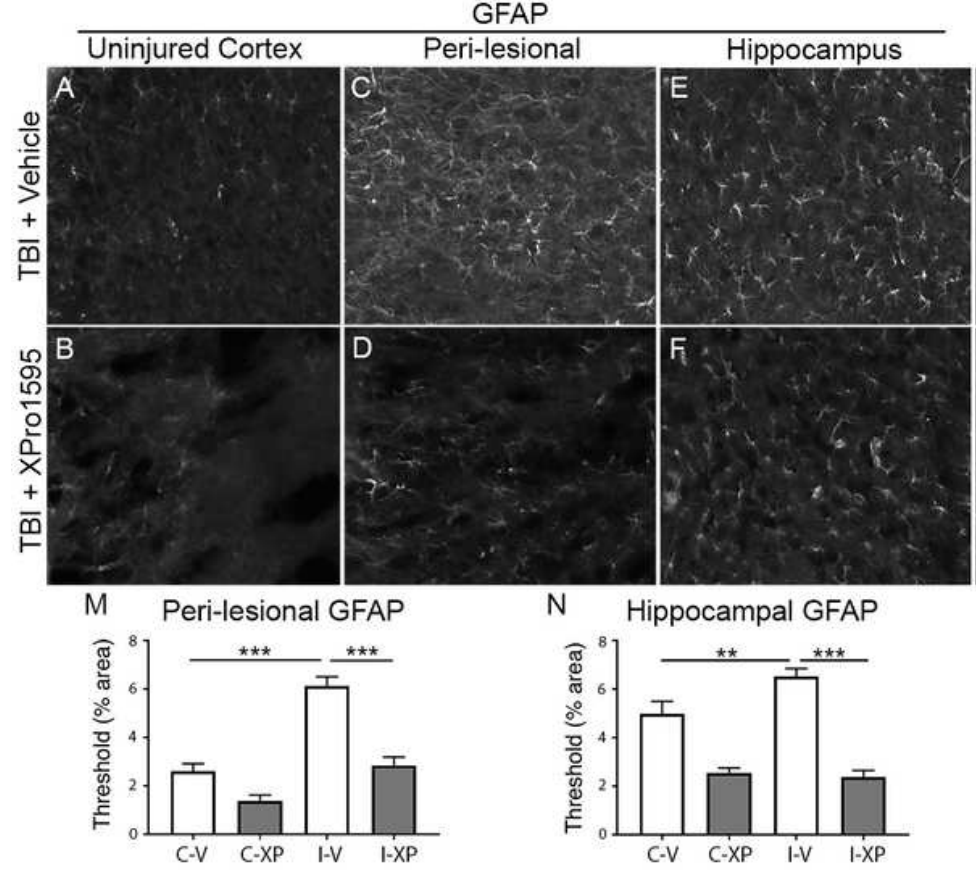
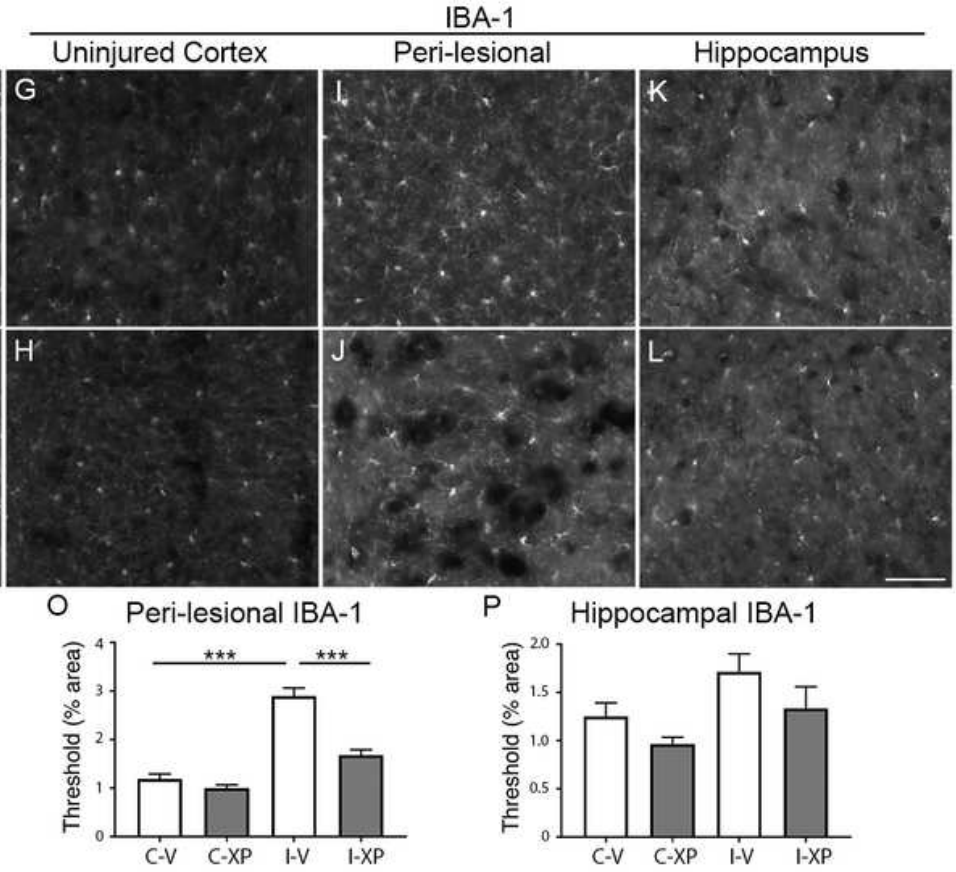

Figure 2

Glial reactivity in the peri-lesional cortex and hippocampus two weeks following TBI. Negligible GFAP (astrocytes) and IBA-1 (microglia/macrophages) protein is expressed in the uninjured cortex $(A, B, G \& H)$, and hippocampus (images not shown), independent of treatment. TBI promotes gliosis in both the perilesional region and hippocampus (C,E,I\&K), although this is prevented by using XPro1595 to neutralize soluble TNF (D, F, J \& L). Graphs show semi-quantitation of glial reactivity in the ipsilateral and 
contralateral hemisphere following TBI (M-P). C-V = contralateral hemisphere+vehicle; C-XP = contralateral hemisphere+XPro1595; I-V = ipsilateral hemisphere+vehicle; I-XP = ipsilateral hemisphere+ XPro1595; TBI+V n=9, TBI+XP $n=9 ; * \star=p<0.01, * \star *=p<0.001$. Scalebar in $L=50 \mu \mathrm{m}$.
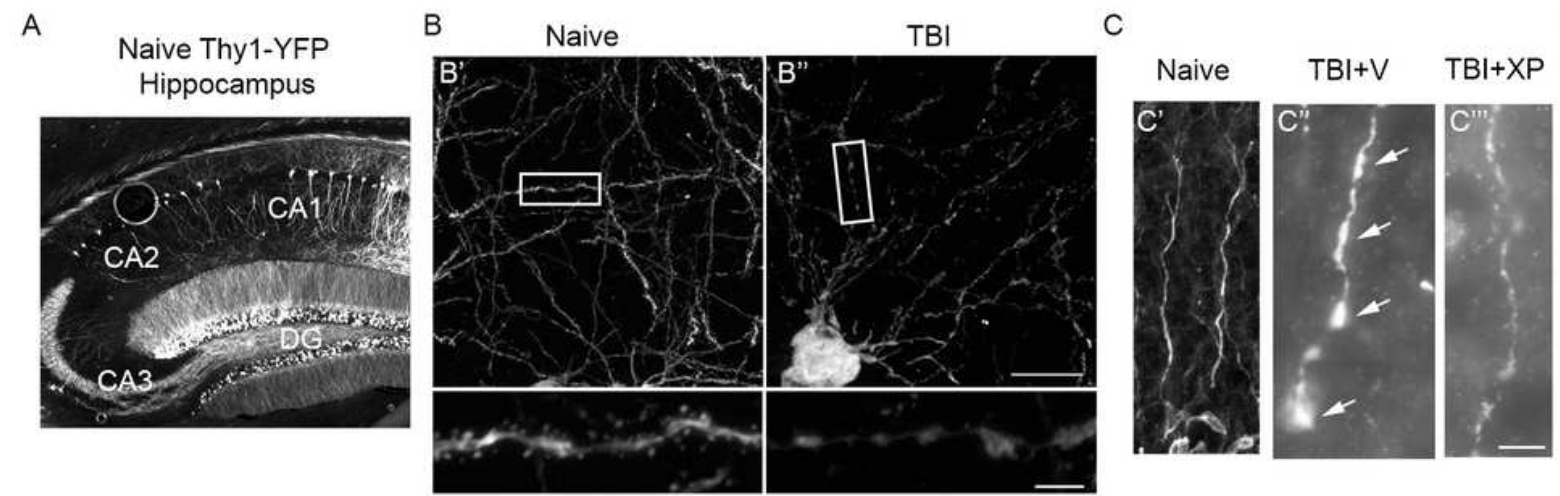

D Dendritic Beads

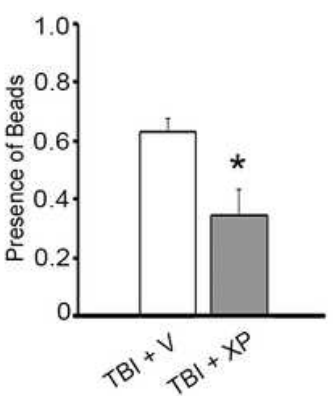

Figure 3

Hippocampal dendritic degeneration following CCI injury. Thy1-YFP-labelled mouse hippocampus showing dentate gyrus (DG), CA1, CA2 and CA3 regions (A). Neuronal dendrites from CA1 region in naive mice show normal morphology ( $\left.B^{\prime}\right)$, while 3 days post-injury CA1 dendrites develop abnormalities ( $\left.B^{\prime \prime}\right)$. $\mathrm{CCl}$-injury appears to reduce spine density, and promotes swellings (beading) along the length of the dendrite (B). In naïve uninjured animals, dendrites from immature neurons within the hippocampal DG that are immunoreactive for doublecortin, show an absence of dendritic swellings ( $\left.C^{\prime}\right)$. However, extensive swellings are observed on immature dendrites of the DG 14 days following the injury ( $\left.C^{\prime \prime}\right)$ that can be significantly reduced when XPro1595 is administered starting 60 minutes post-injury (C'” \&D). V=Vehicle; $\mathrm{XP}=X$ Pro1595; TBI+V n=4, TBI+XP $n=4 ;$ * = p<0.05; Scalebar in B = $20 \mu \mathrm{m}, \mathrm{B}$ inset $=5 \mu \mathrm{m}$, and $\mathrm{C}=2 \mu \mathrm{m}$.

\section{A PSD-95 Expression B Dendrite Morphology}

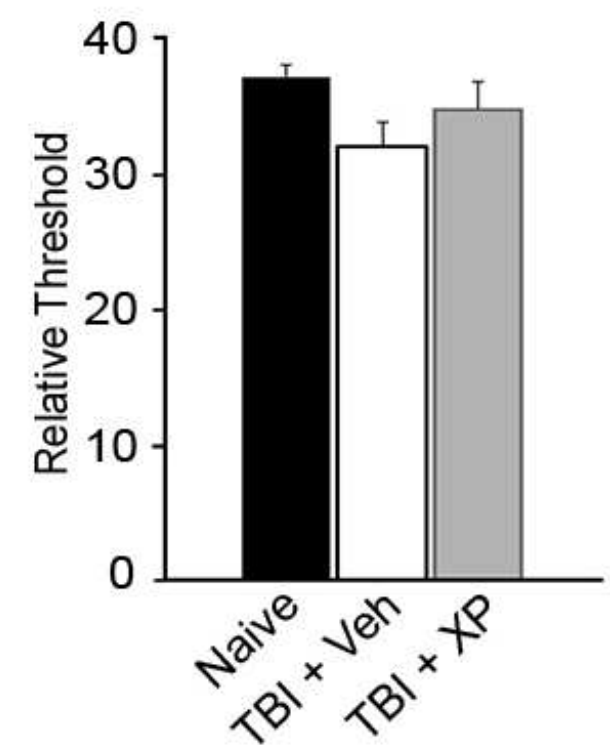

C Spine Density

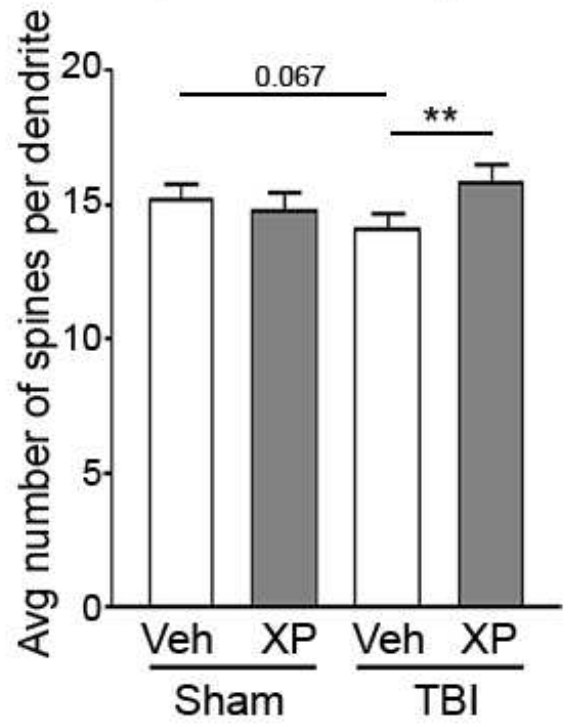

Figure 4 
TBI regulates hippocampal dendritic plasticity via soluble TNF activity. The ipsilateral hippocampus was semi-quantitated for PSD-95 expression; a prominent post-synaptic scaffolding protein, 3 days post-CCI injury. Western blot analysis reveals injury reduced hippocampal PSD-95 expression (A), which was prevented in mice treated with XPro1595 one-hour post-injury. (B) Morphology of Golgi-stained hippocampal slices from sham- and CCl-injured mice, treated with either XPro1595 or Vehicle show changes in spine density. Golgi-stained dendrites were quantitated for spine density (number of spines on a dendrite $200 \mu \mathrm{m}$ in length), revealing that injury reduces dendritic spine density (C), which is prevented in the XPro1595-treated injured mice. Veh=Vehicle, $X P=X P r o 1595 ;(A) n=3$ per group, (C) Sham+Veh $n=7$, Sham $+X P n=5, T B I+V e h n=10, T B I+X P n=10 ; * \star=p<0.01$. Scalebar in $B=2 \mu m$.

A

MWM: Time to Find Platform

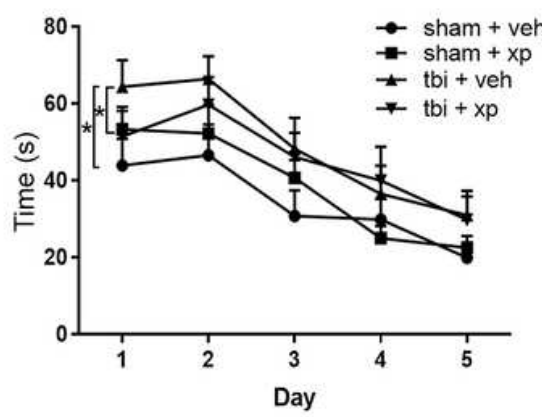

E

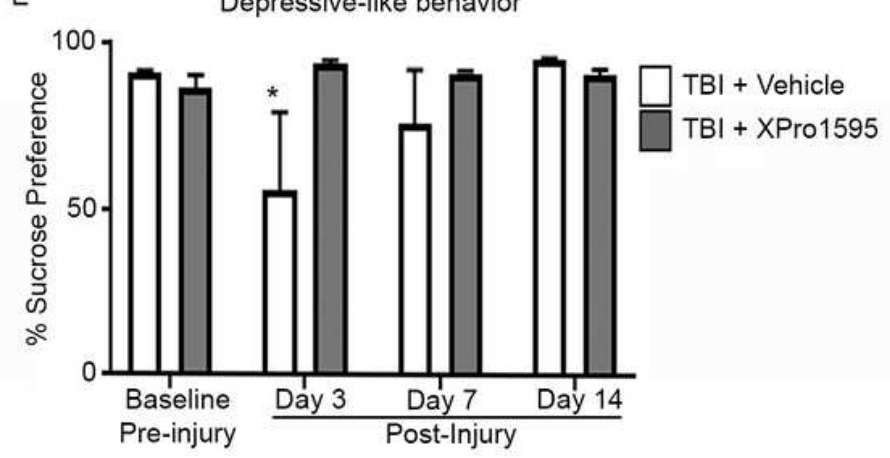

B

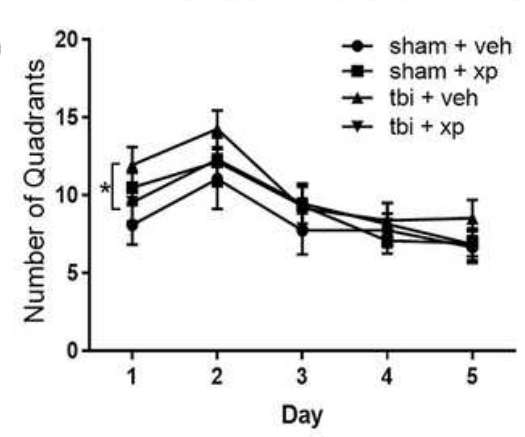

$\mathrm{F}$
MWM Probe:

$\begin{array}{ccc}\text { MWM Probe: } & \text { D } \\ \text { Time in platform quadrant } & \text { \# times to enter platform }\end{array}$

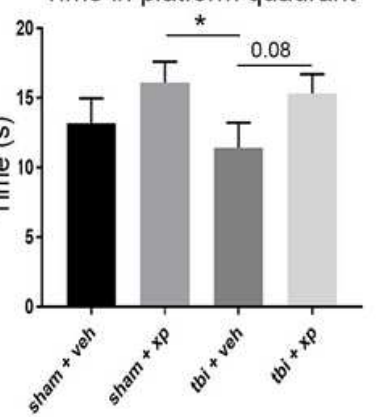
\# times to enter platform quadrant

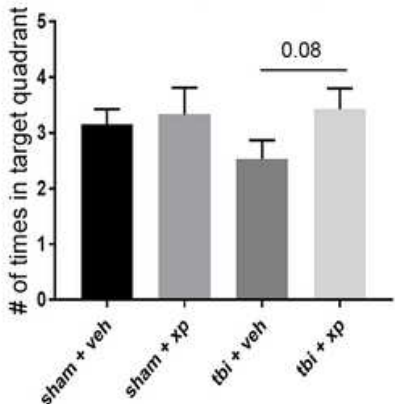

Hindpaw Mechanical Hypersensitivity

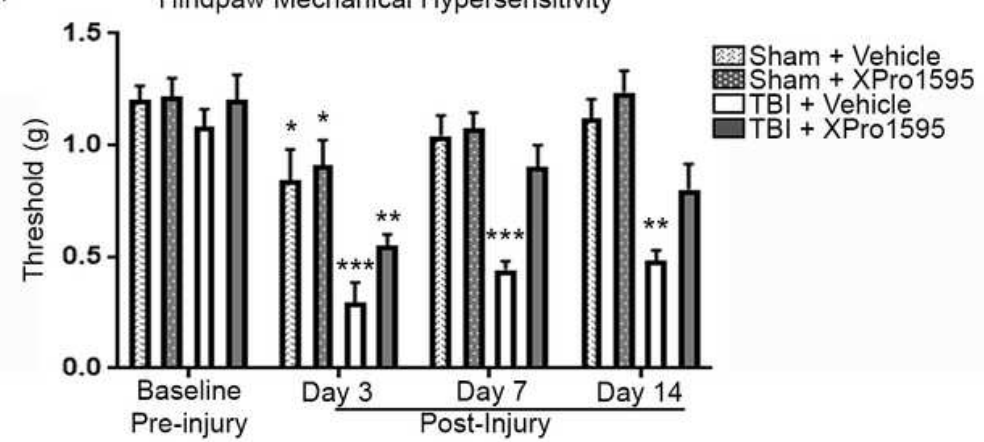

Figure 5

TBI-induced solTNF promotes hippocampal-associated functional impairments. Sham- or CCl-injured mice, treated with vehicle or XPro1595 (starting 60 minutes post-injury) were assessed on spatial learning and memory in the Morris Water Maze (MWM) for 5 consecutive days (post-injury days 7-11). (A\&B) The vehicle-treated injured mice took significantly longer on day 1 to learn to find the platform, by entering significantly more quadrants. Conversely, XPro1595-treated injured mice were able to find the platform in the same amount of time, entering the same number of quadrants, compared to sham-injured mice (independent of treatment). (C\&D) Three days after the last training session (14 days post-injury) the mice were assessed on remembering the location of the platform (probe test: platform removed). Mice with TBI spent less time in the platform quadrant than sham-injured mice, and a tendency to enter the platform quadrant less than sham-injured mice. XPro1595-treated injured mice spent an equal amount of time in the platform quadrant compared to sham-injured mice, and an equal amount of entries into the platform quadrant. (E) Mice were assessed using the sucrose preference test prior to injury (baseline), 
and on post-injury days 3,7 and 14 . Prior to injury the mice preferred to drink the $2 \%$ sucrose water approximately $90 \%$ of the time. Comparatively, vehicle-treated injured mice displayed a temporary reduction in their preference for the sucrose water, which was not observed in the XPro1595-treated injured mice. (F) Mice were assessed on their level of hindpaw mechanical hypersensitivity prior to injury (baseline), and on post-injury days 3, 7 and 14. The sham-injured mice displayed a significant increase in hypersensitivity (reduced threshold) 3 days post-injury, that returns to baseline after the first week. Conversely, the vehicle-treated injured mice display increased hypersensitivity that persists until the end of testing at post-injury day 14 . While the XPro1595-treated injured mice display significantly more hypersensitivity 3 days post-injury, this effect is only temporary, similar to the sham-injured mice. Veh = vehicle; $X P=X P r o 1595 ;(A-D)$ Sham + Veh $n=13$, Sham $+X P n=12, T B I+V e h n=15, T B I+X P n=14 ;(E)$ $T B I+V e h n=4, T B I+X P n=5 ;(F)$ Sham+Veh $n=10$, Sham+XP n=11, TBI+Veh $n=5, T B I+X P n=4 ;$ * = 716 $p<0.05,{ }^{* *}=p<0.01,{ }^{* * *}=P<0.001$. 